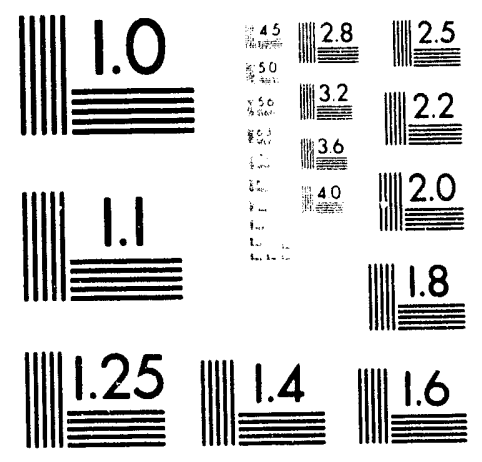



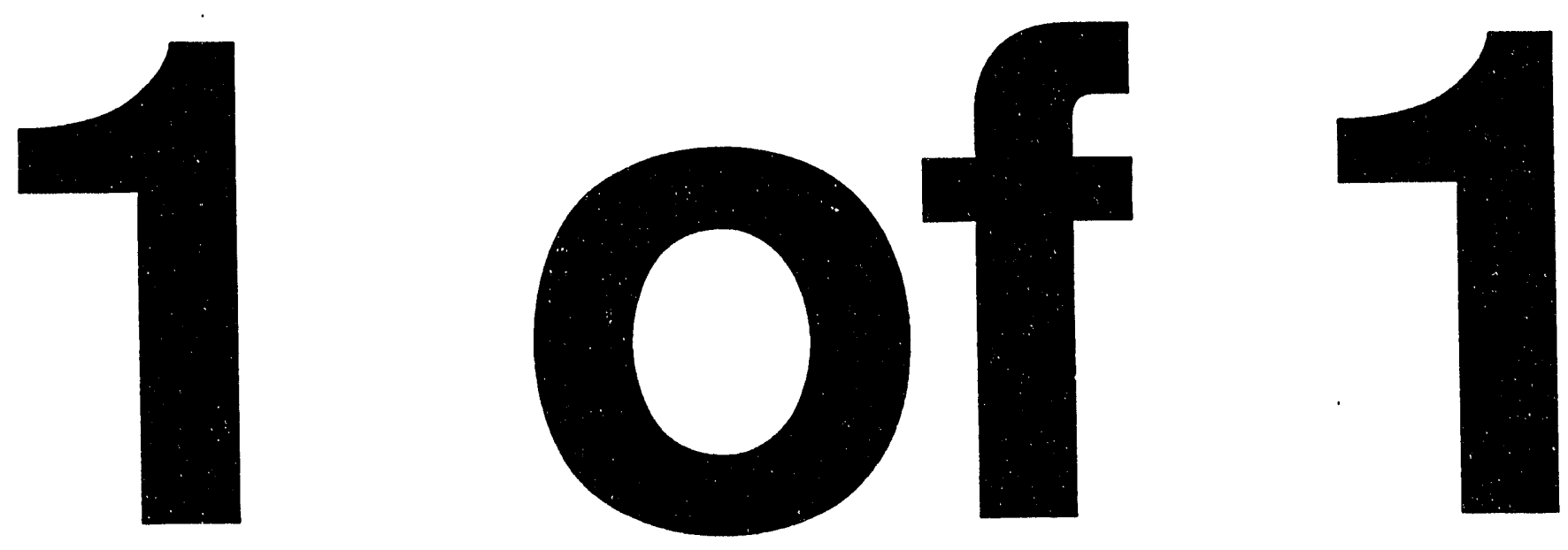
草

SSCL-Preprint-407

May 1993

Distribution Category: 400

E. Tacconi

C. Christiansen

A Wide Range and High Speed Automatic Gain Control

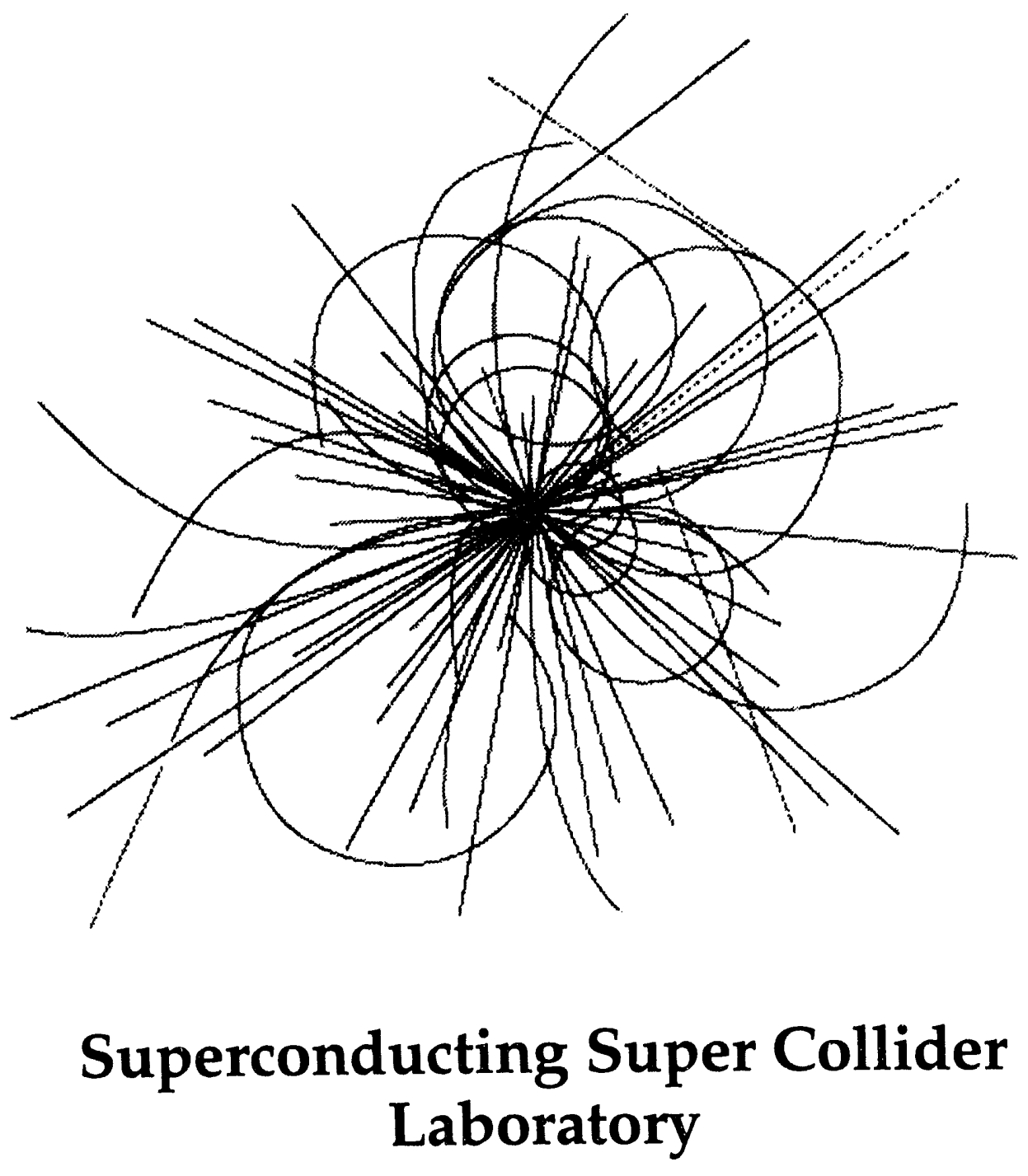




\section{Disclaimer Notice}

This report was prepared as an account of work sponsored by an agency of the United States Government. Neither the United States Government or any agency thereot, nor any of their employees, makes any warranty, express or implied, or assumes any legal liability or responsibility for the accuracy, completeness, or usefulness of any information, apparatus, product, or process disclosed, or represents that its use would not infringe privately owned rights. Reference herein to any specific commercial product, process, or service by trade name, trademark, manufacturer, or otherwise, does not necessarily constitute or imply its endorsement, recommendation, or favoring by the United States Government or any agency thereot. The views and opinions of authors expressed herein do not necessarily state or reflect those of the United States Government or any agency thereof.

Superconducting Super Collider Laboratory is an equal opportunity employer. 


\title{
A Wide Range and High Speed Automatic Gain Control ${ }^{*}$
}

\author{
E. Tacconi and C. Christiansen \\ Superconducting Super Collider Laboratory ${ }^{\dagger}$ \\ 2550 Beckleymeade Ave. \\ Dallas, TX 75237
}

May 1993

*Presented at the 1993 IEEE Particle Accelerator Conference on May 17-20, Washington, D.C. 


\title{
A Wide Range and High Speed Automatic Gain Control
}

\author{
Eugenio J. Tacconi ${ }^{\dagger}$ and Carlos F. Christiansen ${ }^{\dagger}$ \\ Superconducting Super Collider Laboratory* \\ 2550 Beckleymeade Avenue \\ Dallas, Texas 75237
}

\section{Abstract}

Automatic gain control (AGC) techniques have been largely used s nice the beginning of electronics, but in most of the applications the dynamic response is slow compared with the carrier frequency. The problem of developing an automatic gain control with high dynamic response and wide control range simultaneously is analyzed in this work. An ideal gain control law, with the property that the total loop gain remains constant independent of the carrier amplitude, is obtained. The resulting AGC behavior is compared by computer simulations with a linear multiplier AGC. The ideal gain control law can be approximated using a transconductance amplifier. A practical circuit that has been used at CERN in the radio frequency loops of the Booster Synchrotron is presented. The circuit has high speed and 80-dB gain control range.

\section{INTRODUCTION}

In standard automatic gain controls the dynamic response is slow compared with the carrier frequency. When it is necessary to develop an AGC presenting simultaneously high dynamic response and wide control range, some stability problems arise due to the inherent non-linear behavior of the control loop.

A simplified schematic diagram $c f$ an AGC is shown in Figure 1, where $P$ represents the controlled amplifier, $A$ the power amplifier, $D$ the amplitude detector, $F$ a low pass filter, and $C$ a cascade compensator. The $\mathrm{AGC}$ has to regulate the amplitude of the output voltage, $V_{o}$ following the reference voltage, $V_{\text {ref }}$ The amplitude of the carrier voltage, $V_{i n}$, is the main perturbation to be rejected.

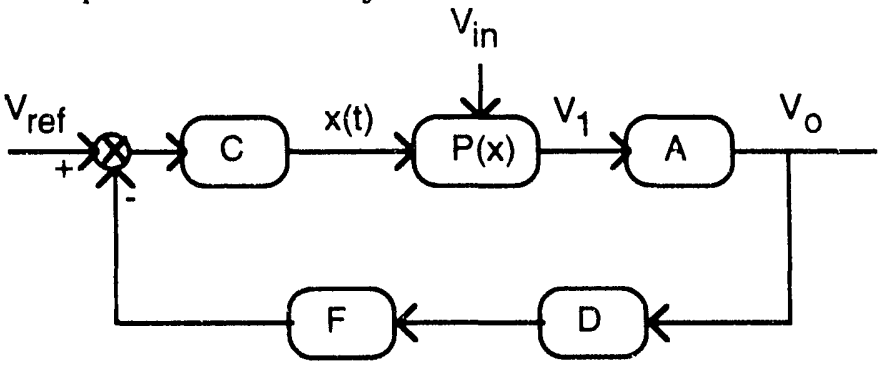

Figure 1. AGC block diagram.

The gain of the controlled amplifier is a function of the control voltage, $x(t)$. Thus, the control system is non-linear, and gain loop and stability are usually dependent on the operating point. The system has to be designed in order to be stable for the

\footnotetext{
* Operated by Universities Research Association, Inc., for the U.S. Department of Energy under Contract No. Dli-AC35-89ER40486.

†On leave from Leici, UNLP, Conicet, Argentina.
}

maximum loop gain, and a poor dynamic behavior is obtained for low-gain operating points. The effect of the gain control law on the loop gain variation range is analyzed in the next section.

\section{GAIN CONTROL LAW}

The output of the variable gain amplifier is given by:

$$
V_{1}=V_{\text {in }} \cdot P(x) \text {. }
$$

Thus, its equivalent gain depends on the operating point and is given by:

$$
p=\frac{d V_{1}}{d x}=V_{\text {in }} \cdot \frac{d P(x)}{d x} .
$$

From Figure 1, the loop gain $L G$ can be expressed by:

$$
L G=C \cdot p \cdot A \cdot F \cdot D .
$$

Expressions (2) and (3) show that the loop gain is usually dependent on the carrier amplitude $\left(V_{i n}\right)$. This is apparent when a linear multiplier type of control amplifier is used.

\section{A. Linear Multiplier}

In this case, the gain control law is:

$$
P(x)=V_{\text {in }} \cdot k \cdot x,
$$

where $k$ is a constant. Equation (3) becomes

$$
L G=C \cdot k \cdot V_{i n} \cdot A \cdot F \cdot D .
$$

Thus, the loop gain is directly proportional to the carrier amplitude. To obtain adequate stability over the whole amplitude range, a low-frequency dominant pole compensation is generally used. Then, when the carrier amplitude is reduced, the AGC constant time is proportionally increased.

The time domain system behavior is shown in Figures 2 and 3, where the input carrier signal and the regulated output for iwo different operaiciso puints are representeci. Figure 2 shows a good dynamic response, obtained when the carrier signal has maximum amplitude. Neverseless, if the carrier signal amplitude is reduced (Figure 3), the AGC constant time is increased, resulting in a slow time-domain response.

The system behavior is highly dependent on the gain control law of the controlled amplifier. Different controlled amplifiers have been proposed in the literature [1], [2]. 


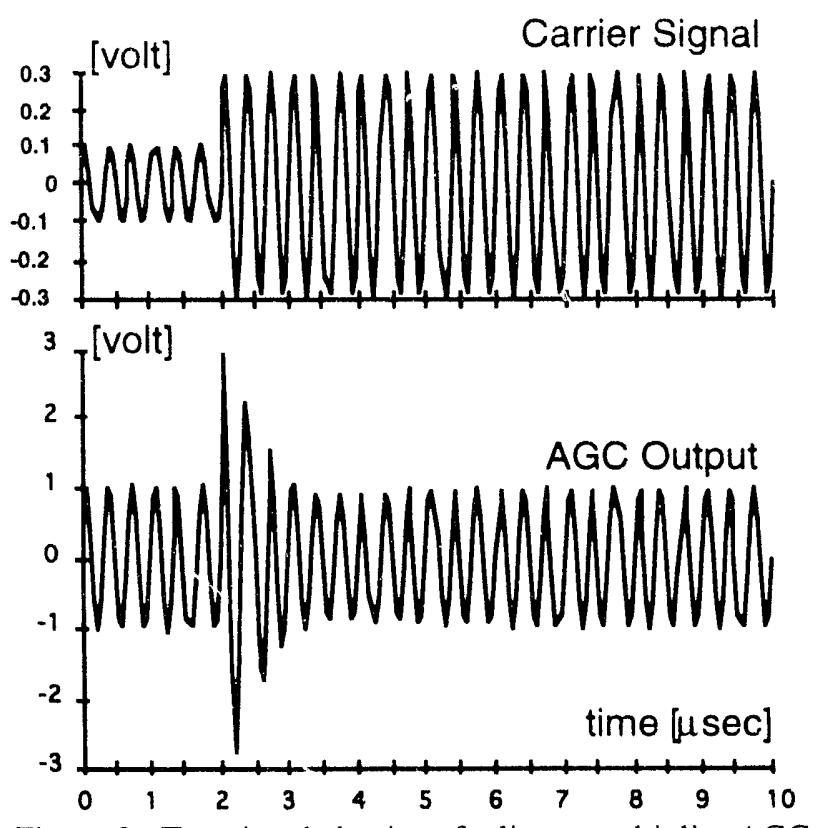

Figure 2. Transient behavior of a linear multiplier AGC Carrier amplitude, $0.1 \mathrm{~V}-0.3 \mathrm{~V}$

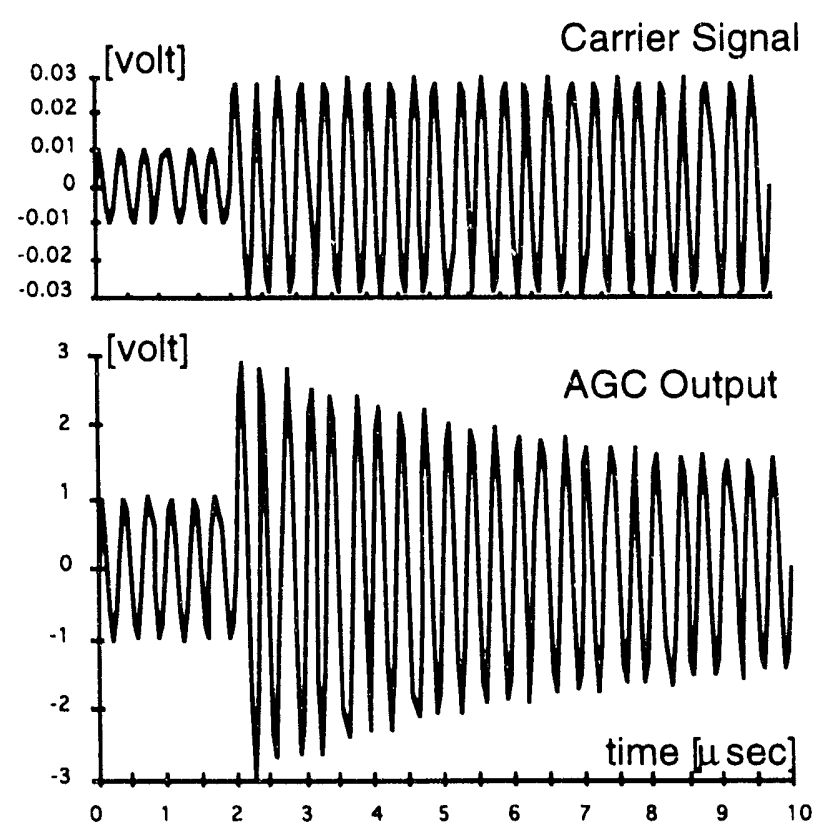

Figure 3. Transient behavior of a linear multiplier AGC. Carrier amplitude, $0.01 \mathrm{~V}-0.03 \mathrm{~V}$

\section{B. Ideal Control Law}

Our aim is to derive a gain control law which gives, for a constant output amplitude, a constant loop gain independent of the carrier amplitude.

The purpose of an AGC is to obtain an output signal with a given constant amplitude. Let us call this output amplitude $\mathrm{V}_{\mathrm{o}} \mathrm{o}^{\mathrm{O}}$

$$
V_{0}^{0}=\left.V_{\text {in }} \cdot P(x) \cdot A\right|_{V_{o}=V_{o}^{o}}=A \cdot V_{1}^{o} .
$$

For a constant $p$, a constant loop gain is obtained:

$$
p=\left.\frac{d V_{1}}{d x}\right|_{V_{1}=V_{1}^{o}}=\frac{V_{1}^{o}}{P(x)} \cdot \frac{d P(x)}{d x}=K_{1},
$$

where $K_{1}$ is an arbitrary constant. From Eqs. (6) and (7), it is

$$
P(x)=K_{2} \cdot \exp \left(\frac{K_{1} \cdot A}{V_{o}^{o}} \cdot x\right)
$$

where $K_{1}$ and $K_{2}$ are arbitrary constants.

Thus, by imposing a loop constant gain, independent of the carrier amplitude, the control law of an ideal controlled gain amplifier has been deduced (expression (8)).

$$
L G=C \cdot K_{1} \cdot A \cdot F \cdot D
$$

An AGC using this control law will present a dynamic behavior that changes with the amplitude value of the output voltage but is independent of the carrier amplitude. The input carrier presents a wide amplitude range, while the regulated outpui amplitude is usually constant.

The computer simulation results of an AGC with an exponential control law are shown in Figures 4 and 5. In order to compare the behavior of both AGCs, the cascade compensator has been designed for having relative stability similar to the case of Figure 2.

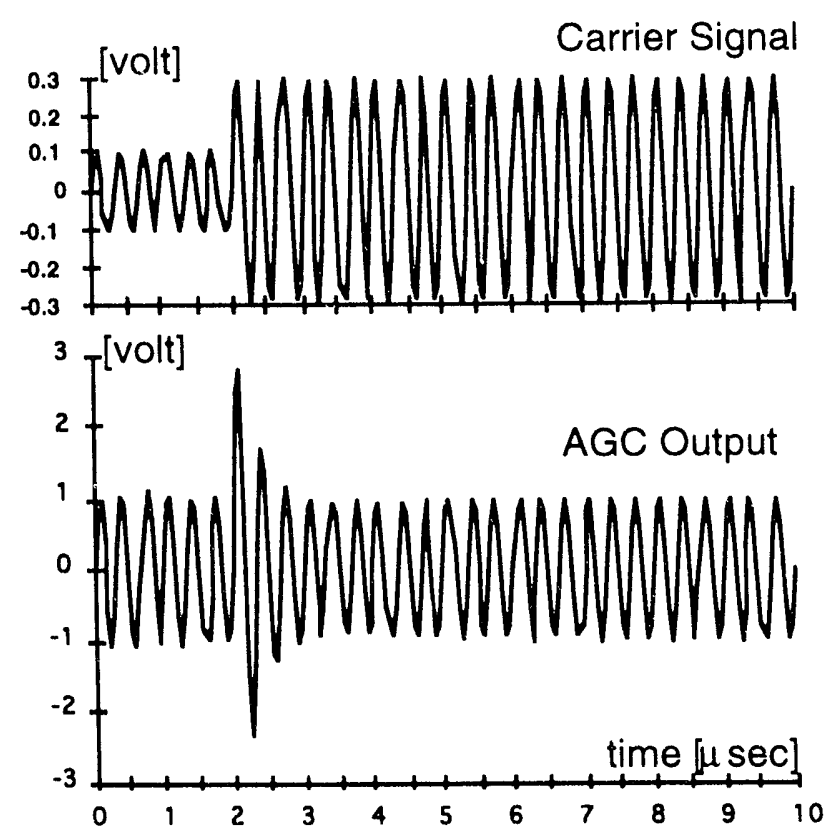

Figure 4. Transient behavior of an exponential law AGC. Carrier amplitude, $0.1 \mathrm{~V}-0.3 \mathrm{~V}$ 

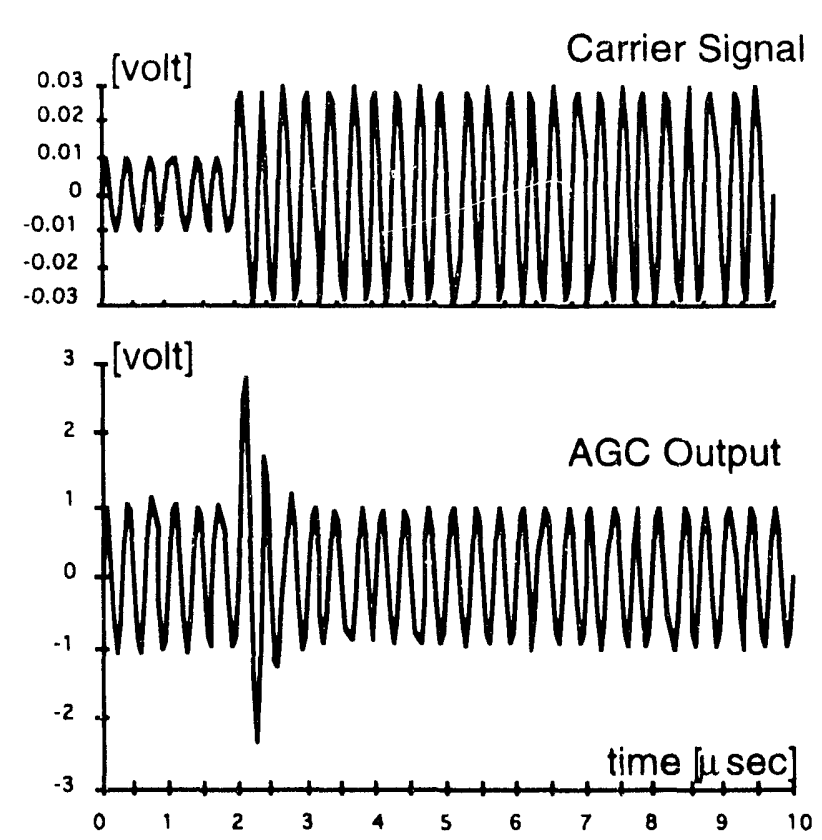

Figure 5. Transient behavior of an exponential law AGC. Carrier amplitude, 0.01V-0.03V

The simulations have been carried out under the following conditions: the power amplifier $A$ and the feedback loop have unitary gain, the amplitude detector is a quadratic detector, the filter $F$ presents a single pole at $0.5 \mathrm{MHz}$, and the compensator $C$ has a dc gain of $10^{4}$ having a single pole at $30 \mathrm{~Hz}$.

Equation 9 shows that the loop gain is constant over the whole AGC range. Thus, the AGC constant time is also constant and independent of the carrier amplitude. This feature is shown in Figures 4 and 5, where the AGC dynamic response continues been the same although the carrier amplitude has been reduced $20 \mathrm{~dB}$. The advantages of the method are clearly seen by comparing Figures 3 and 5 .

\section{CIRCUIT IMPLEMENTATION}

An exponential law gain control can be approximated by a transconductance amplifier [3]. In this case, the gain control - law is $x p r e s s e d$ by:

$$
P(x)=c_{1} /\left[1+\exp \left(\frac{x-c_{2}}{c_{3}}\right)\right]
$$

where $c_{1}, c_{2}$ and $c_{3}$ are constants and $x \geq c_{2}$.

$$
p=-\frac{V_{1}^{o}}{c_{3}}\left[\exp \left(\frac{x-c_{2}}{c_{3}}\right)\right] /\left[1+\exp \left(\frac{x-c_{2}}{c_{3}}\right)\right] .
$$

From Eqs. (3) and (11), the loop gain variation is limited to less than $6 \mathrm{~dB}$ over the whole control range. If the AGC range is $40 \mathrm{~dB}$, the $3-\mathrm{dB}$ corner frequency changes 100 times for a linear multiplier AGC and less than 2 times for a transconductance amplifier AGC. Thus, by using a transconductance amplifier, an AGC with a dynamic behavior almost independent of the carrier amplitude is obtained.
An AGC having these characteristics has been designed and implemented at CERN for the radio frequency loops of the Booster Synchrotron. For this application, it was necessary to regulate a radio frequency signal with an amplitude range of $80 \mathrm{~dB}$ and frequencies of $3-9 \mathrm{MHz}$. The $\mathrm{AGC}$ requirements included minimum phase rotation and fast $\mathrm{AGC}$ time response. The problem was solved by using two AGCs in cascade connection. Each AGC presents a control range of $40 \mathrm{~dB}$ and a constant time of less than $1 \mathrm{~s}$ [4]. The circuit was implemented with a commercial transconductance amplifier gain controller (MC1590) having a 60-dB AGC range. The amplitude detector is a full-wave mean value rectifier, the passive filter $F$ presents a corner frequency at $8 \mathrm{kHz}$, and the cascade compensator $C$ is a dc amplifier with a single-pole at $1 \mathrm{MHz}$. The total open loop transfer function has a dc gain of 50, and the closed loop bandwidth is $0.4 \mathrm{MHz}$.

\section{CONCLUSIONS}

An automatic gain control having simultaneously a high dynamic response and a wide control range has been analyzed in this work. It has been deduced that an exponential control law AGC presents a constant loop gain, independent of the carrier amplitude. The computer simulation results show the advantages of the method. For practical applications, the exponential gain control can be approximated with a transconductance amplifier. For designing a high-speed AGC, a transconductance-controlled gain amplifier must be considered over other commercial AGCs. The characteristics of an AGC of this type, used in the radio frequency loops of the Booster Synchrotron at CERN, are also presented.

\section{REFERENCES}

[1] D. V. Mercy, "A Review of Automatic Gain Control Theory," The Radio and Electronic Engineer, Vol. 51, No. 11/12, pp. 479-590, (1981).

[2] I. M. Filanovsky and V. A. Piskarev, "Automatic Gain Control by Differential Pair Current Splitting," Int. Journal Electronics, Vol. 62, No. 2, pp. 243-250, (1987).

[3] Brent Trout, "A High Gain Integrated Circuit RF-IF Amplifier with Wide Range AGC," Application Note AN-513, Motorola.

[4] E. J. Tacconi, "Amplificateur a Large Gamme Dynamique, Fiable Bruit et Commande Automatique de Gain pour le Systeme de Controle RF du Faisceau PSB,". CERN PS/BR No. 85-3, (1985). 

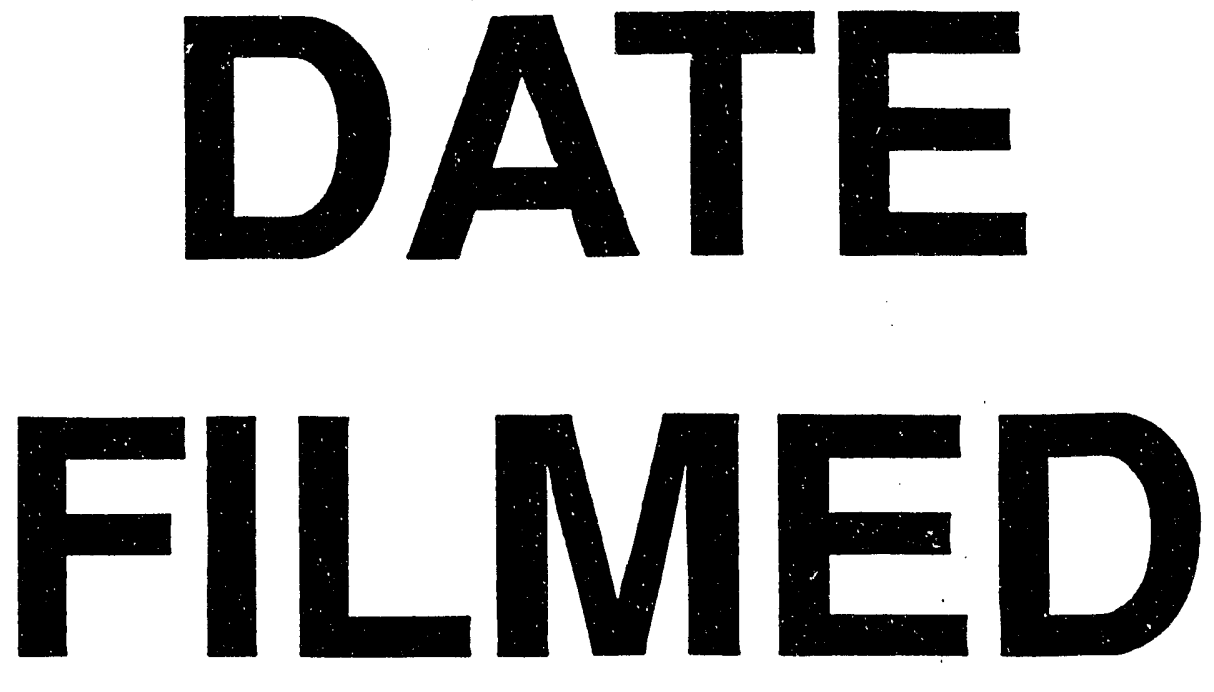

$11 / 12 / 93$
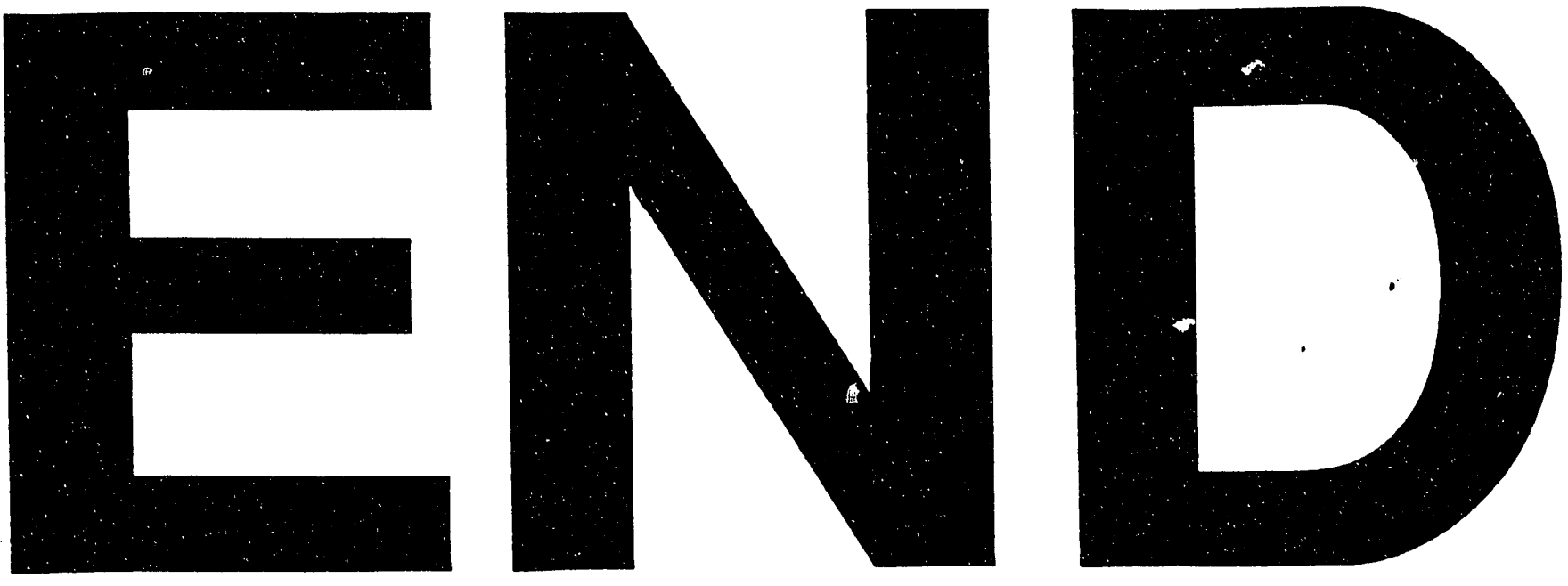
